\title{
A Summary of the Consensus for the Management of Thyroid Disorders in Malaysia
}

\author{
Suehazlyn Zainudin ${ }^{1}$, Zanariah Hussein ${ }^{2}$, Mohammad Yazid Jalaludin ${ }^{3}$, Wong Ming ${ }^{1}$, \\ Lim Shueh Lin ${ }^{4}$, Malik Mumtaz ${ }^{5}$, Rokiah Pendek ${ }^{3}$, Mafauzy Mohamed ${ }^{6}$, Amir Khir ${ }^{7}$ \\ ${ }^{1}$ Faculty of Medicine, National University Malaysia, Kuala Lumpur, Malaysia \\ ${ }^{2}$ Putrajaya Hospital, Putrajaya, Malaysia \\ ${ }^{3}$ Faculty of Medicine, University Malaya Medical Centre, Petaling Jaya, Malaysia \\ ${ }_{4}^{4}$ Penang General Hospital, Penang, Malaysia \\ ${ }^{5}$ Island Hospital, Penang, Malaysia \\ ${ }^{6}$ School of Health Sciences, Hospital Universiti Sains Malaysia, Kota Baru, Kelantan, Malaysia \\ 'Penang Medical College, Penang, Malaysia
}

\begin{abstract}
Thyroid disorders are amongst one of the most common endocrine diseases in Malaysia. In 1998, a taskforce consisting of experts in the field of thyroidology was set up to develop consensus recommendations for the management of thyroid disorders in the country based on available scientific evidence at that point in time. The following article summarizes the recommendations put together by the task force incorporating some of the recent available evidence with regards to the treatment of thyroid disorders.
\end{abstract}

Keywords: consensus recommendations, Malaysia, thyroid disorders

\section{Introduction}

The consensus recommendations for the management of thyroid disorders was jointly developed by the Malaysia Endocrine Metabolism Society (MEMS), Academy of Medicine Malaysia and the Ministry of Health Malaysia in 1998. The aim of the consensus was to provide clinical guidelines on the diagnosis and management of common thyroid disorders in the country. The majority of the thyroid disorders in the country are managed by primary care doctors and few cases were referred to the tertiary centers. It was therefore prudent that a guideline that is easily understood and implementable was developed. The guideline which was published in 2000 is currently in the process of revision.

\section{Summary of methodology of guidelines development}

The committee responsible for the development of the guideline consisted of experts in the field of thyroidology from the above three organizations. The guidelines were divided into sections; each section representing broad categories of thyroid disorders. Each section was delegated to two or three individual experts who then collected literature relevant to the subject matter. Relevant keywords and terms were used to generate MEDLINE searches for scientific literature, focussing on peerreviewed articles. The various sections were compiled and reviewed individually by all members of the committee.

ISSN 0857-1074

Printed in the Philippines

Copyright (C) 2012 by the JAFES

Received May 8, 2012. Accepted May 15, 2012.
Recommendations were graded based on the levels of evidence as Grade A to C, based on the strength of the scientific data.

\section{Summary of recommendations}

\section{Thyrotoxicosis}

Thyrotoxicosis is a clinical state of excess thyroid hormones due to various etiologies. Although Graves' disease (GD) is the most common cause, the prevalence of toxic multinodular goitre increases with age and in areas of iodine deficiency. Other known causes include toxic adenoma, thyroiditis, factitious intake of thyroxine and ovarian diseases. The signs and symptoms of thyrotoxicosis varies according to individuals and only moderately correlate with the level of thyroid hormone. ${ }^{1}$

To confirm the diagnosis, both free T4 (fT4) and serum thyrotrophin / thyroid stimulating hormone (TSH) should be measured. Free T4 measurement is preferred to total T4 measurement as the latter is affected by protein binding abnormalities. In patients with normal fT4 but suppressed TSH, free T3 (fT3) measurements are indicated to diagnose patients with T3-toxicosis; a condition is thought to represent an early stage of the disease or caused by an autonomous nodule. Thyroid scan may be useful in suspected cases of thyroiditis or toxic adenoma. A recent history of iodine exposure is important as radioiodine uptake may be low within 1-2 months' exposure to

Corresponding author: Suehazlyn Zainudin, $M D$

Division of Diabetes \& Endocrinology,

National University of Malaysia Medical Centre,

Jalan Yaacob Latif, Bandar Tun Razak, Cheras,

56000 Kuala Lumpur, Malaysia

Telephone number: $+603-91456958$

E-mail address: suehazlyn@gmail.com 
iodinated contrast or an iodine-rich diet, or amiodarone treatment. In nodular thyroid, technetium scintigraphy may also be useful in determining the cause of thyrotoxicosis and subjects the patient to lesser total body radiation exposure. TRAb measurements may be used for diagnosis of GD, and differentiating thyroiditis from factitious thyroxine intake.

Three modalities of treatment are available for patients with thyrotoxicosis: antithyroid drugs (ATD), surgery and radioactive iodine therapy. A quality-of-life study found no long-term differences amongst patients who had any one of the three treatments. ${ }^{2}$ Thionamides (methimazole [MMZ], carbimazole [CMZ] or prophylthiouracil [PTU]) are the most commonly used ATDs. ATDs are favored in patients with high likelihood of remission, the elderly or those with high surgical risks or limited life expectancy, nursing home individuals or those unable to follow radiation safety regulations, patients with history of neck surgery or irradiation and patients with moderate to severe graves' opthalmopathy. ATDs should be maintained for 12-18 months and then tapered off. A remission rate of over $50 \%$ has been reported in patients, especially females with mild disease, small goitres and negative TRAb. ${ }^{3}$ Men, smokers, large goitres and high titres of TRAb are associated with lower remission. In patients with symptomatic thyrotoxicosis, beta-blockers are indicated in heart rate more than $90 \mathrm{bpm}$, especially in the elderly and those with concomitant heart disease. In those with contraindications to beta-blockade, oral verapamil and diltiazem may be used.

The indications and contraindications for RAI therapy are shown in Table 1. A fixed dose approach with dosing between $370 \mathrm{MBq}$ to $555 \mathrm{MBq}$ is recommended. ${ }^{5}$ Adverse effects are rare. The risk of eventual hypothyroidism is high, especially after treatment of GD. There can be transient exacerbation of symptoms. A potential problem is progression of GO. ${ }^{6}$ In selected patients, a course of oral prednisone at $0.4-0.5 \mathrm{mg} / \mathrm{kg}$ daily, starting 2-3 days after RAI for 1 month and gradually reduced reduces the risk of this complication. Early thyroxine replacement 2 weeks after RAI may reduce the progression of GO. ${ }^{7}$ Hypothyroidism rates within the first year are very much dependent on the dose of RAI. Thereafter, the incidence is 2 to 3 percent per year. ${ }^{8}$

Surgery is indicated when medical therapy fails, in goiters with pressure effects, suspected thyroid malignancy and in those with severe progressive opthalmopathy.

Complications of thyrotoxicosis include thyroid cardiomyopathy, hypokalaemic periodic paralysis, pretibial myxoedema and Grave's opthalmopathy (GO). Thyroid cardiomyopathy occurs in long-standing poorly controlled thyrotoxicosis, especially in the elderly. Rapid atrial fibrillation is treated with digoxin and beta-blockers, whilst anticoagulation is recommended in the presence of cardiac dilatation, cardiac thrombus or valve abnormalities. Patients who develop hypokalaemic periodic paralysis may require potassium replacement and spironolactone until euthyroidism achieved whereas pretibial myxoedema may respond to local steroid application under occlusive dressing.

Table 1. Indications and contraindications for RAI therapy

The main indications for RAI therapy include the following conditions

Hyperthyroidism

Graves' disease

Toxic multinodular goitre or

Hyperfunctioning thyroid nodules

The issue of treating children with RAI remains controversial

Non-toxic multinodular goitre

Thyroid cancer.

Contra-indications for RAI therapy

Pregnancy

Breast feeding

Severe uncontrolled thyrotoxicosis

The mode of therapy for Grave's opthalmopathy (GO) is dependent upon clinical activity score (CAS) and severity. Active but non-severe GO is treated symptomatically with artificial tears and taping the eye. Moderate to severe active GO is treated with glucocorticoids. There is controversy surrounding the best mode, dose and duration of corticosteroid. IV methylprednisolone is reported to be superior to oral prednisolone in response rate with fewer side effects. ${ }^{9}$ The recommended course is $0.5 \mathrm{~g}$ of IV methylprednisolone once weekly for 6 weeks, followed by $0.25 \mathrm{~g}$ weekly for 6 weeks. ${ }^{10}$ In sight threatening GO, IV glucocorticoid may be given over shorter duration at a higher dose (1-3g per week). Surgical decompression should be considered in cases that do not respond. Radiotherapy has been used in GO with overall response rate of $60 \%$. It is most effective on soft tissue inflammation and recent extra-ocular muscle involvement.

\section{Thyroid crisis}

Thyrotoxic crisis, or thyroid storm, carries a high mortality rate if early diagnosis is not made and treated. No specific laboratory tests are confirmatory and the diagnosis is primarily clinical. Burch and Wartofsky ${ }^{11}$ had published a scoring system assessing the degree of dysfunction in various systems which include thermoregulatory, central nervous system, gastrointestinal and cardiovascular.

Adequate hydration and treatment of any precipitating factors are essential. Hyperthermia needs to be controlled aggressively with tepid sponging, cooling blanket and acetaminophen. The use of aspirin or NSAIDS is contraindicated. Specific therapies used are directed against the thyroid. Beta-blocker, namely propanolol 60 $80 \mathrm{mg}$ every 4 to 6 hourly, is administered to achieve adequate control of heart rate. Thionamides (PTU 200mg 
or MMZ every 4 to 6 hourly) block thyroid hormone synthesis within one to two hours after administration. Lugol's iodine 10 drops 8 hourly are used to block the release of thyroid hormones from the gland. However, it should be given 1 hour after thionamide administration to prevent it from being used as a substrate for new thyroid hormone synthesis. Intravenous dexamethasone 1 to $2 \mathrm{mg}$ 6 hourly or intravenous hydrocortisone 100mg 8 hourly, reduces $\mathrm{T} 4$ to $\mathrm{T} 3$ conversion. Glucocorticoids are also useful in treating potentially associated adrenal insufficiency. Rarely, plasmapheresis has been used in life-threatening cases or in patients with contraindications to thionamides that require urgent correction of hyperthyroidism prior to surgery. Iodide therapy and glucocorticoids can be stopped once the patient's condition stabilises (usually after 3-5 days).

\section{Hypothyroidism}

Hypothyroidism occurs due to deficiency of thyroid hormones resulting in a hypometabolic state, commonly manifesting as a slowing in physical and mental activity. Individuals may be asymptomatic or commonly have subtle, nonspecific symptoms.

To confirm diagnosis, TSH and fT4 levels should be measured. In primary hypothyroidism, fT4 levels are low with elevated serum TSH levels. Serum TSH may be mildly elevated in patients with normal serum fT4 levels indicating subclinical hypothyroidism. In central hypothyroidism serum fT4 levels are low with low or normal serum TSH levels.

Treatment is aimed at rendering the patient clinically and biochemically euthyroid. The usual starting dose of Lthyroxine for an adult is 50 or $100 \mathrm{ug} /$ day increasing to a maintenance dose of 100-200ug/day. Thyroxine should be administered preferably in the early morning, prior to food to aid absorption. Alternatively it can be taken at bedtime.

In patients with ischaemic heart disease (IHD), gross hypothyroidism and in the elderly, the usual starting dose of L-thyroxine is $25 \mathrm{ug} /$ day. The dosage should be increased slowly (ie. every 2-4 weeks) according to the patient's response. Thyroid function tests should be routinely evaluated 4 to 6 weeks after dose adjustment. If angina occurs, reduce to previous dosage or withhold treatment temporarily, while management of IHD is optimised.

\section{Subclinical hypothyroidism}

An estimated $2 \%$ to $5 \%$ of patients with subclinical hypothyroidism (SCH) progress to overt hypothyroidism annually. Annual monitoring of TSH in untreated patients is indicated. Decision to treat SCH will depend on the risk of progression to overt hypothyroidism such as the degree of TSH elevation and anti-TPO antibody titre elevation.

\section{Monitoring in hypothyroidism}

Serum TSH measurement is useful in assessing adequacy of thyroxine replacement therapy in primary hypothyroidism. The target TSH is $0.5-3.0 \mathrm{mU} / \mathrm{l}$. In the elderly, target TSH is in the higher normal range. Measurements of serum TSH and fT4 should be done 8 12 weeks after initiation of therapy to determine the maintenance dose and subsequently every 6 months to 1 year. Inadequate thyroxine replacement may occur when there is decreased thyroxine absorption, accelerated thyroxine disposal or increased thyroxine binding with estrogen use. TSH is only useful as a measure of thyroid disease if the hypothalamic-pituitary-thyroid axis is intact. When pituitary or hypothalamic disease is suspected, fT4 measurement is preferred and is targeted in the upper half of normal range.

\section{Myxoedema coma}

Myxoedema coma is a rare, severe and life-threatening complication of hypothyroidism. Urgent treatment is required because mortality rates are high $(20 \%-50 \%)$. Treatment should be initiated if there is a high index of clinical suspicion without waiting for laboratory results. Patients may present with hypotension, hypoglycaemia, respiratory failure, hypothermia and hyponatremia.

Treatment requires prompt administration of thyroid hormone, intensive care monitoring and supportive care. L-thyroxine $300-400 \mathrm{ug}$ is given orally or parenterally if available. Alternatively, small doses of tri-iodothyronine (10ug 8 hourly IV or orally) may be used. Hypotension may respond to crystalloid infusion, vasopressive agents or with thyroid hormone replacement. Mechanical ventilation is indicated for patients with diminished respiratory drive or obtundation. Underlying infections need to be treated. Hypothermia requires gradual rewarming measures; aggressive rewarming may lead to peripheral vasodilatation and hypotension. Hyponatremia is treated with water restriction; however, hypertonic saline may be indicated. Metabolism of sedatives, narcotics, and anesthetics may be slowed significantly, and should therefore be avoided. Intravenous hydrocortisone should be given until patient regains consciousness, as associated adrenal dysfunction is common

\section{Congenital hypothyroidism}

Congenital hypothyroidism (CHT) is a congenital endocrine disorder resulting from inadequate thyroid hormone for the metabolic needs of a newborn infant. Most infants are asymptomatic at birth. The presence of prolonged jaundice, poor feeding, constipation or an unusually quiet baby should alert the clinician to the possibility of hypothyroidism. Ideally screening should be done on heel prick blood 2-5 days after birth. Screening of newborn with cord blood TSH is an acceptable alternative. Those with high cord blood TSH levels would be recalled 
for confirmation of diagnosis by measurement of TSH and $\mathrm{fT}_{4}$ using venous blood at 3-7 days of age.

Treatment should commence as soon as the diagnosis is made with L-thyroxine is $10-15 \mathrm{mcg} / \mathrm{kg} / \mathrm{dose}$ daily to normalize $\mathrm{fT}_{4}$ within 2 weeks and TSH within 1 month. Serum $\mathrm{fT}_{4}$ should be kept at the upper half of the reference range in the first year of life, with serum TSH within the reference range. With early adequate thyroid replacement therapy, these infants are expected to have normal intellectual, growth and development.

Serum $\mathrm{fT}_{4}$ and $\mathrm{TSH}$ measurements should be performed at 2 and 4 weeks after the initiation of L-thyroxine; every 1 to 2 months during the first 6 months of life; every 3 to 4 months between 6 months and 3 years; every 6 to 12 months until growth is completed; and at more frequent intervals when compliance is questioned, abnormal values are obtained, or dose or source of medication has been changed. Treatment is life-long except in children suspected of having transient hypothyroidism whereby therapy is temporarily stopped for 4 to 6 weeks at 3 years of age for re-evaluation.

\section{Single thyroid nodule}

The prevalence of thyroid nodules in Malaysia is not known, but in iodide-replete areas it is in the region of $5 \%$ for females and $1 \%$ for males. ${ }^{12}$ Thyroid cancer occurs in $5 \%$ of nodules found by palpation alone in the general population ${ }^{13}$ and up to $15 \%$ in high risk individuals.

Serum TSH is a useful first step in the investigation. A suppressed TSH points to a functioning/hot nodule and should be followed by a thyroid scintiscan. A hyperfunctioning nodule is rarely malignant. In patients with normal TSH, a diagnostic ultrasound scan (USS) should be performed followed by fine needle aspiration cytology (FNAC) if indicated. Sonographic features that increase suspicion of malignancy are hypoechogenicity, microcalcifications, increased intranodular blood flow on Colour Doppler, absence of halo, irregular margins and anterioposterior - to-transverse diameter $(\mathrm{A} / \mathrm{T})$ ratio $>1$. Small lesions, cystic lesions with suspicious solid component and lesions where previous FNAC have been non-diagnostic are best done via USS guidance rather than by palpation. ${ }^{14}$ In benign FNAC, continuous surveillance is recommended as a change in the nodule size may point to the need for repeat FNAC.

\section{Conclusion}

The 1998 thyroid consensus was developed with the aim of providing a much needed guide for all practitioners involved in management of common thyroid disorders. Practitioners are advised to use this consensus coupled with their own sound judgment based on the patients' clinical presentation and other data available at any point in time. Updating of the consensus with newer evidence is currently ongoing and will be made available to all practitioners once ready.

\section{References}

1. Trzepacz PT, Klein I, Roberts M, et al. Graves' disease; An analysis of thyroid hormone levels and hyperthyroid signs and symptoms.Am J Med.1989;87:558-561

2. Abraham-Nording M, Torring $\mathrm{O}$, Hamberger B, et al. Graves' disease: A long-term quality-of-life follow up of patients randomized to treatment with antithyroid drugs, radioidine or surgery. Thyroid. 2005;15:1279-1286

3. Laurberg P, Buchholtz Hansen PE, Iversen E, et al. Goitre size and outcome of medical treatment of Graves' disease. Acta Endocrinol (Copenh).1986;111:39-43

4. Amit Allahabadia, Daykin J, Sheppard MC, Gough SCL, Franklyn JA. Radioiodine treatment of hyperthyroidism-prognostic factors for outcome. J Clin Endocrinol Metab 2001; 86(8): 3611-3617

5. Meier DA. Procedure guidelines for therapy of thyroid disease with iodine-131 (Sodium Iodide) J Nucl Med 2002; 43; 856-861

6. Torring O, Tallstedt L, Wallin G et al. The Thyroid Study Group Graves' hyperthyroidism: Treatment with antithyroid drugs, surgery, or radioiodine-a prospective, randomized study. J Clin Endocrinol Metab 1996; 81:2986-2993

7. Perros P, Kendall-Taylor P, Neoh C, Frewin S, Dickinson J. A prospective study of the effects of radioiodine therapy for hyperthyroidism in patients with minimally active Graves' Ophthalmopathy. J Clin Endocrinol Metab 2005; 90:5321-5323

8. Aizawa, Y, Yoshida, K, Kaise, N, et al. The development of transient hypothyroidism after iodine-131 treatment in hyperthyroid patients with Graves' disease: Prevalence, mechanism and prognosis. Clin Endocrinol 1997; 46:1.

9. Zang S, Ponto KA, Kahaly GJ .Intravenous glucocorticoids for Graves' orbitopathy: Efficacy and morbidity J Clin Endocrinol Metab.2011.96:320-332.

10. Bartalena L, Pinchera A, Marcocci C. Management of Graves' ophthalmopathy: Reality and perspective. Endo Reviews.2000. 21:168-199.

11. Burch HB, Wartofsky L. Life-threatening thyrotoxicosis. Thyroid storm. Endocrinol Metab Clin North Am. Jun 1993;22(2):263-77

12. Tunbridge WMG, Evered DC, Hall R, et al. The spectrum of thyroid disease in a community: The Whickham Survey. Clin Endocrinol (Oxf ). 1977. 7:481-493.

13. Mazzaferri EL. Management of a solitary thyroid nodule. N Engl J Med. 1993.328:553-559.

14. Carmeci C, Jeffrey RB, McDougall IR, Nowels KW, Weigel RJ. Ultrasound-guided fine-needle aspiration biopsy of thyroid masses.1998. Thyroid 8:283-289

The 2000 Consensus for the Management of Thyroid Disorders in Malaysia is currently undergoing revision. Requests for further details on the recommendations shall be forwarded to the Malaysian Endocrine and Metabolic Society (MEMS). 